\title{
SESHADRI CONSTANTS ON SOME QUOT SCHEMES
}

\author{
CHANDRANANDAN GANGOPADHYAY, KRISHNA HANUMANTHU, \\ AND RONNIE SEBASTIAN
}

\begin{abstract}
Let $E$ be a vector bundle of rank $n$ on $\mathbb{P}^{1}$. Fix a positive integer $d$. Let $\mathcal{Q}(E, d)$ denote the Quot scheme of torsion quotients of $E$ of degree $d$ and let $\operatorname{Gr}(E, d)$ denote the Grassmann bundle that parametrizes the $d$-dimensional quotients of the fibers of $E$. We compute Seshadri constants of ample line bundles on $\mathcal{Q}(E, d)$ and $\operatorname{Gr}(E, d)$.
\end{abstract}

\section{INTRODUCTION}

Let $X$ be a projective variety over an algebraically closed field $k$ and let $L$ be a nef line bundle on $X$. For a point $x \in X$, the Seshadri constant of $L$ at $x$ is defined as

$$
\varepsilon(X, L, x):=\inf _{x \in C} \frac{L \cdot C}{\operatorname{mult}_{x} C},
$$

where the infimum is taken over all curves in $X$ passing through $x$. Here $L \cdot C$ denotes the intersection multiplicity and mult $_{x} C$ denotes the multiplicity of $C$ at $x$. When there is no confusion about $X$, to simplify notation we denote $\varepsilon(X, L, x)$ by $\varepsilon(L, x)$.

Seshadri constants were introduced by Demailly [Dem92] as a way to tackle the Fujita Conjecture. He was motivated by an ampleness criterion of Seshadri [Har70, Theorem I.7.1]. These constants turned out to be important invariants associated to projective varieties. As an illustration, let $X$ be a Fano variety of dimension $N$ and let $K_{X}$ denote the canonical bundle on $X$. Then $X$ is isomorphic to $\mathbb{P}^{N}$ if and only if there exists a smooth point $x \in X$ such that $\varepsilon\left(X,-K_{X}, x\right)>N$; see [BS09, LZ18]. A lot of research is currently focused on studying Seshadri constants.

Seshadri constants on surfaces have been the primary focus of researchers, but some results are known in general only for special classes of varieties. For example, several results are known in the case of Fano varieties [Lee03], abelian varieties [Laz96, Nak96, Bau98] or toric varieties [Ito14].

In this paper, we compute Seshadri constants for ample line bundles on Quot schemes over $C:=\mathbb{P}^{1}$. Let $E$ be a vector bundle of rank $n$ on $C$

Date: October 14, 2021.

2010 Mathematics Subject Classification. 14C20.

Key words and phrases. Seshadri constants, Quot schemes, projective bundles, Grassmann bundles.

The second author was partially supported by a grant from Infosys Foundation and DST SERB MATRICS grant MTR/2017/000243. 
and let $d$ be a positive integer. Let $\mathcal{Q}(E, d)$ denote the Quot scheme of torsion quotients of $E$ of degree $d$. The Nef cone of $\mathcal{Q}(E, d)$ is described in [GS20]. In [Str87] the author restricts his attention to the case when $E$ is the trivial bundle, but considers more general Quot schemes (not just torsion quotients). We use this description to compute the Seshadri constants of ample line bundles on $\mathcal{Q}(E, d)$.

When $E$ is the trivial bundle of rank $n$ we will also denote $\mathcal{Q}(E, d)$ by $\mathcal{Q}$ or $\mathcal{Q}(n, d)$. In Section 2, we consider the Quot scheme $\mathcal{Q}(n, d)$ where $d>1$. The Nef cone is generated by naturally defined line bundles $\left[\mathcal{L}_{d-1}\right]$ and $\left[\Phi^{*} \mathcal{O}_{\mathbb{P}^{d}}(1)\right]$ (see discussion preceding (2.4) for notation and more details). The main result of Section 2 is the following (see Definition 2.11 for the definition of the closed subset $\mathcal{Z}$ and the open set $\mathcal{U}=\mathcal{Q} \backslash \mathcal{Z})$.

Theorem 1.1. Fix integers $a, b>0$. If $x \in \mathcal{U} \subset \mathcal{Q}$ we have

$$
\varepsilon\left(a \cdot\left[\mathcal{L}_{d-1}\right]+b \cdot\left[\Phi^{*} \mathcal{O}_{\mathbb{P}^{d}}(1)\right], x\right)=a .
$$

If $x \in \mathcal{Z}=\mathcal{Q} \backslash \mathcal{U}$ we have

$$
\varepsilon\left(a \cdot\left[\mathcal{L}_{d-1}\right]+b \cdot\left[\Phi^{*} \mathcal{O}_{\mathbb{P}^{d}}(1)\right], x\right)=\min \{a, b\} .
$$

In Section 3, we deal with the case $\mathcal{Q}^{\prime}:=\mathcal{Q}(E, d)$ where $E$ is an arbitrary vector bundle and $d>1$. The Nef cone is generated by line bundles $\left[\mathcal{L}_{L, \mathcal{Q}^{\prime}}\right]$ and $\left[\Phi^{\prime *} \mathcal{O}_{\mathbb{P} d}(1)\right]$, (see discussion preceding (3.5) for notation and more details). There is a natural inclusion $j: \mathcal{Q}^{\prime} \hookrightarrow \mathcal{Q}$. The main result of Section 3 is the following.

Theorem 1.2. Assume $d>1$. Fix integers $a, b>0$. If $x \in \mathcal{Q}^{\prime}$ such that $j(x) \in \mathcal{U}$, then we have

$$
\varepsilon\left(a \cdot\left[\mathcal{L}_{L, \mathcal{Q}^{\prime}}\right]+b \cdot\left[\Phi^{\prime *} \mathcal{O}_{\mathbb{P}^{d}}(1)\right], x\right)=a .
$$

If $x \in \mathcal{Q}^{\prime} \backslash \mathcal{U}$ we have

$$
\varepsilon\left(a \cdot\left[\mathcal{L}_{L, \mathcal{Q}^{\prime}}\right]+b \cdot\left[\Phi^{\prime *} \mathcal{O}_{\mathbb{P}^{d}}(1)\right], x\right)=\min \{a, b\} .
$$

The case $d=1$ corresponds to projective bundles. This is dealt with in Theorem 4.3. Finally, using similar methods we compute Seshadri constants on Grassmann bundles over $C$ in Section 5, see Theorem 5.2.

If $L$ is an ample line bundle on an $N$-dimensional projective variety $X$, we have $0<\varepsilon(X, L, x) \leq \sqrt[N]{L^{N}}$ for all $x \in X$. So we define the following:

$$
\begin{gathered}
\varepsilon(X, L, 1):=\sup _{x \in X} \varepsilon(L, x), \text { and } \\
\varepsilon(X, L):=\inf _{x \in X} \varepsilon(L, x) .
\end{gathered}
$$

While $\varepsilon(X, L)$ can be arbitrarily small by an example of Miranda [Laz04, Example 5.2.1], it is conjectured that we always have $\varepsilon(X, L, 1) \geq 1$ when $k$ is the field of complex numbers; see [Laz04, Conjecture 5.2.4]. This is known to be true when $X$ is a surface (see [EL93]), but in higher dimension it is open, in general. As a consequence of our main results, we conclude that. 
Corollary 1.3. The inequality $\varepsilon(X, L, 1) \geq 1$ holds for ample line bundles $L$ when $X$ is $\mathcal{Q}$ or $\mathcal{Q}^{\prime}$.

\section{SeshadRi COnstants on $\mathcal{Q}(n, d)$}

Throughout we assume the base field to be an algebraically closed field $k$. In this section, we consider the Quot scheme associated to the trivial bundle. The results proved in this section will be used when we consider the general case in Section 3.

Let $C:=\mathbb{P}^{1}$. Fix integers $n, d \geq 1$. Let $\mathcal{Q}:=\mathcal{Q}(n, d)$ be the Quot scheme of torsion quotients of the vector bundle $\mathcal{O}_{C}^{n}$ of degree $d$. It is well known that $\mathcal{Q}$ is a smooth projective variety of dimension $n \cdot d$. Let $\mathcal{O}_{C \times \mathcal{Q}}^{n} \rightarrow \mathcal{B} \rightarrow 0$ be the universal quotient over $C \times \mathcal{Q}$. This quotient has the following universal property: Let $T$ be a finite type scheme over $k$. Suppose we have a quotient $\mathcal{O}_{C \times T}^{n} \rightarrow B_{T} \rightarrow 0$ over $C \times T$ such that $B_{T}$ is flat over $T$ and for all closed points $t \in T$, the sheaf $\left.B_{T}\right|_{C \times t}$ is a torsion sheaf of degree $d$. Then there exists a unique map $f_{T}: T \rightarrow \mathcal{Q}$ such that

$$
\left(i d_{C} \times f_{T}\right)^{*}\left[\mathcal{O}_{C \times \mathcal{Q}}^{n} \rightarrow \mathcal{B}\right]=\mathcal{O}_{C \times T}^{n} \rightarrow B_{T} .
$$

In this section we compute Seshadri constants of ample line bundles on $\mathcal{Q}$. We begin by describing the Nef cone of $\mathcal{Q}$.

Let $p_{C}: C \times \mathcal{Q} \rightarrow C$ and $p_{\mathcal{Q}}: C \times \mathcal{Q} \rightarrow \mathcal{Q}$ be the projections. The sheaf $p_{\mathcal{Q}_{*}}(\mathcal{B})$ is a vector bundle over $\mathcal{Q}$ of rank $d$. Define the line bundle

$$
\mathcal{O}_{\mathcal{Q}}(1):=\operatorname{det}\left(p_{\mathcal{Q}_{*}}(\mathcal{B})\right) \text {. }
$$

Let

$$
\Phi: \mathcal{Q} \rightarrow S^{d} \mathbb{P}^{1} \cong \mathbb{P}^{d}
$$

be the Hilbert-Chow map (see [GS19, Section 2]). This map has the following pointwise description. If $\left[\mathcal{O}_{C}^{n} \rightarrow B\right]$ is a torsion quotient of degree $d$, then $\Phi$ maps this to the point in $S^{d} \mathbb{P}^{1}$ corresponding to $\sum_{x \in \operatorname{Supp}(B)} l\left(B_{x}\right)[x]$, where $l\left(B_{x}\right)$ denotes the length of the $\mathcal{O}_{C, x}$-module $B_{x}$.

The Néron-Severi space $N^{1}(\mathcal{Q})$ is two-dimensional and the classes $\left[\mathcal{O}_{\mathcal{Q}}(1)\right]$ and $\left[\Phi^{*} \mathcal{O}_{\mathbb{P}^{d}}(1)\right]$ form a basis (see [GS20, Corollary 3.10]). Define

$$
\mathcal{L}_{d-1}:=\mathcal{O}_{\mathcal{Q}}(1) \otimes \Phi^{*} \mathcal{O}_{\mathbb{P}^{d}}(d-1) .
$$

The nef cone $\operatorname{Nef}(\mathcal{Q}) \subset N^{1}(\mathcal{Q})$ of $\mathcal{Q}$ is the cone generated by the following classes (see [GS20, Proposition 6.1])

$$
\operatorname{Nef}(\mathcal{Q})=\mathbb{R}_{\geq 0}\left[\mathcal{L}_{d-1}\right]+\mathbb{R}_{\geq 0}\left[\Phi^{*} \mathcal{O}_{\mathbb{P}^{d}}(1)\right] .
$$

For a line bundle $L$ on $C$ we denote

$$
\mathcal{L}_{L, \mathcal{Q}}:=\operatorname{det}\left(p_{\mathcal{Q} *}\left(\mathcal{B} \otimes p_{C}^{*} L\right)\right) .
$$

Lemma 2.6. Given any point $x \in \mathcal{Q}$ there exists a curve $L_{1, x} \cong \mathbb{P}^{1} \hookrightarrow \mathcal{Q}$ passing through $x$ such that $\left[\mathcal{L}_{d-1}\right] \cdot\left[L_{1, x}\right]=1$ and $\left[\Phi^{*} \mathcal{O}_{\mathbb{P}^{d}}(1)\right] \cdot\left[L_{1, x}\right]=0$. 
Proof. Let $E=\mathcal{O}_{C}^{n}$. Let $x \in \mathcal{Q}$ correspond to the quotient $x: E \rightarrow B \rightarrow 0$. Fix a quotient $B \rightarrow B^{\prime} \rightarrow 0$ where $B^{\prime}$ is a torsion sheaf of degree $d-1$. Let $A$ be the kernel of $E \rightarrow B \rightarrow 0$ and let $A^{\prime}$ be the kernel of the composition $E \rightarrow B \rightarrow B^{\prime} \rightarrow 0$. Then $A \subset A^{\prime} \subset E$ and we have an exact sequence

$$
0 \rightarrow A^{\prime} / A \rightarrow B \rightarrow B^{\prime} \rightarrow 0 .
$$

Hence, $A^{\prime} / A$ is a torsion sheaf of degree 1 , that is, $A^{\prime} / A \cong k_{c}$, where $k_{c}$ is the skyscraper sheaf with fibre $k$ at a point $c \in C$. Let $A_{c}^{\prime}$ be the fiber of the sheaf $A^{\prime}$ over the point $c \in C$ and let $Z:=\mathbb{P}\left(A_{c}^{\prime}\right)$. Let $p_{1}: C \times Z \rightarrow C$ and $p_{2}: C \times Z \rightarrow Z$ be the projections. Let $i: c \times Z \hookrightarrow C \times Z$ be the inclusion. Then we define a quotient on $C \times Z$ as the composition

$$
p_{1}^{*} A^{\prime} \rightarrow i_{*} i^{*} p_{1}^{*} A^{\prime} \cong i_{*}\left(A_{c}^{\prime} \otimes \mathcal{O}_{Z}\right) \rightarrow i_{*} \mathcal{O}_{Z}(1) .
$$

Let $A_{Z} \subset p_{1}^{*} A^{\prime} \subset p_{1}^{*} E$ be the kernel of the above composition. Let us denote the quotient $p_{1}^{*} E / A_{Z}$ by $B_{Z}$. Then we have an exact sequence on $C \times Z$

$$
0 \rightarrow i_{*} \mathcal{O}_{Z}(1) \rightarrow B_{Z} \rightarrow p_{1}^{*} B^{\prime} \rightarrow 0 .
$$

Hence $B_{Z}$ is flat over $Z$ such that $\left.B_{Z}\right|_{C \times z}$ is a torsion sheaf of degree $d$ for every $z \in Z$. Therefore the quotient $p_{1}^{*} E \rightarrow B_{Z} \rightarrow 0$ defines a map $f: Z \rightarrow \mathcal{Q}$. It is clear that $x \in \mathcal{Q}$ is in the image of this map. Using [GS20, Lemma 3.1 (ii)] and (2.8) we get that $f^{*} \mathcal{O}_{\mathcal{Q}}(1)=\mathcal{O}_{Z}(1)$. Let $z \in Z$. Then the quotient corresponding to $f(z)$ sits in the short exact sequence,

$$
\left.0 \rightarrow k_{c} \rightarrow B_{Z}\right|_{z} \rightarrow B^{\prime} \rightarrow 0,
$$

obtained by restricting (2.8) to $C \times z$. This shows that the $\operatorname{divisor} \operatorname{div}\left(\left.B_{Z}\right|_{z}\right)$ corresponding to $\left.B_{Z}\right|_{z}$ is the sum $\operatorname{div}\left(B^{\prime}\right)+c$. Thus, $\Phi \circ f$ is constant; that is, the image of $f$ is contained in a fiber of $\Phi$.

Next we will show that $f$ is a closed immersion. Note that $E$ is globally generated. Let $V:=H^{0}(C, E)$. Then we have a surjection $V \otimes \mathcal{O}_{C} \rightarrow E$. This gives surjective maps

$$
V \otimes p_{C}^{*} \mathcal{O}_{C} \rightarrow p_{C}^{*} E \rightarrow \mathcal{B} .
$$

Letting $K$ denote the kernel of the map $V \otimes p_{C}^{*} \mathcal{O}_{C} \rightarrow \mathcal{B}$ and observing that $\left.K\right|_{q}$ decomposes into a direct sum of line bundles $\mathcal{O}_{C}\left(b_{i}\right)$ with $-d \leq b_{i} \leq 0$, one easily checks that $R^{1} p_{\mathcal{Q} *}\left(K \otimes p_{C}^{*} \mathcal{O}(d-1)\right)=0$. Let $L:=\mathcal{O}_{C}(d-1)$. Tensoring (2.9) with $p_{C}^{*} L$, applying $p_{\mathcal{Q} *}$ and taking exterior power, we see that

$\operatorname{det}\left(p_{\mathcal{Q} *}\left(\mathcal{B} \otimes p_{C}^{*} L\right)\right)=\mathcal{L}_{L, \mathcal{Q}}$ is a globally generated line bundle on $\mathcal{Q}$.

In [GS20, Proposition 6.1] it is proved that

$$
\mathcal{L}_{L, \mathcal{Q}} \cong \mathcal{O}_{\mathcal{Q}}(1) \otimes \Phi^{*} \mathcal{O}_{\mathbb{P}^{d}}(d-1)=\mathcal{L}_{d-1}
$$

Since $\Phi \circ f$ is constant, it follows that $f^{*} \mathcal{L}_{L, \mathcal{Q}} \cong f^{*} \mathcal{O}_{\mathcal{Q}}(1) \cong \mathcal{O}_{Z}(1)$. Consider the composite map $Z \rightarrow \mathcal{Q} \rightarrow \mathbb{P}^{N}$, where the second map is given by $\mathcal{L}_{L, \mathcal{Q}}$. Since the pullback of $\mathcal{O}_{\mathbb{P}^{N}}(1)$ along this map is $\mathcal{O}_{Z}(1)$ it follows that the composite, and so also $f$, is a closed immersion. 
Let $L_{1, x} \subset Z \subset \mathcal{Q}$ denote any line passing through $x$. Then it is clear that $L_{1, x}$ satisfies the assertions of the lemma.

Let $\mathcal{O}_{C \times \mathcal{Q}}^{n} \rightarrow \mathcal{B}$ denote the universal quotient on $C \times \mathcal{Q}$. Pushing this forward we get a map $\mathcal{O}_{\mathcal{Q}}^{n} \rightarrow p_{\mathcal{Q} *} \mathcal{B}$ of sheaves on $\mathcal{Q}$. Let $\mathcal{F}$ denote the cokernel. From Grauert's Theorem it is clear that for $q \in \mathcal{Q}$ the fiber $\mathcal{F} \otimes k(q)$ is the cokernel of the map $H^{0}\left(C, \mathcal{O}_{C}^{n}\right) \rightarrow H^{0}\left(C, \mathcal{B}_{q}\right)$. Note that the image of this map cannot be 0 , thus, $\operatorname{dim}(\mathcal{F} \otimes k(q)) \leq d-1$ for all points $q \in \mathcal{Q}$. The set

$$
\{q \in \mathcal{Q} \mid \operatorname{dim}(\mathcal{F} \otimes k(q)) \geq d-1\}=\{q \in \mathcal{Q} \mid \operatorname{dim}(\mathcal{F} \otimes k(q))=d-1\}
$$

is a closed subset.

Definition 2.11. Define $\mathcal{Z} \subset \mathcal{Q}$ to be the closed set consisting of points $q$ for which the image of $H^{0}\left(C, \mathcal{O}_{C}^{n}\right) \rightarrow H^{0}\left(C, \mathcal{B}_{q}\right)$ is 1-dimensional. Define $\mathcal{U}:=\mathcal{Q} \backslash \mathcal{Z}$.

Lemma 2.12. Given any point $x \in \mathcal{Z}$ there exists a curve $L_{2, x} \cong \mathbb{P}^{1} \hookrightarrow \mathcal{Q}$ passing through $x$ such that $\left[\mathcal{L}_{d-1}\right] \cdot\left[L_{2, x}\right]=0$ and $\left[\Phi^{*} \mathcal{O}_{\mathbb{P}^{d}}(1)\right] \cdot\left[L_{2, x}\right]=1$.

Proof. Let $x \in \mathcal{Z}$. Let $0 \neq w \in H^{0}(C, B)$ be an element in the image of the map $H^{0}\left(C, \mathcal{O}_{C}^{n}\right) \rightarrow H^{0}(C, B)$. Then the quotient corresponding to $x$ factors as $\mathcal{O}_{C}^{n} \stackrel{v}{\rightarrow} \mathcal{O}_{C} \stackrel{w}{\rightarrow} B$. Associated to the surjection $v: \mathcal{O}_{C}^{n} \rightarrow \mathcal{O}_{C}$ we have a section $\eta_{v}: \mathbb{P}^{d} \hookrightarrow \mathcal{Q}$ of $\Phi$ as in [GS20, Equation (3.12)] which passes through the point $x$ such that

$$
\eta_{v}^{*} \mathcal{L}_{d-1}=\eta_{v}^{*}\left(\mathcal{O}_{\mathcal{Q}}(1) \otimes \Phi^{*} \mathcal{O}_{\mathbb{P}^{d}}(d-1)\right) \cong \mathcal{O}_{\mathbb{P}^{d}} .
$$

This is explained in the second paragraph of the proof in [GS20, Proposition 6.1]. Now choose any line in $\mathbb{P}^{d}$ such that its image under $\eta_{v}$, call it $L_{2, x}$, passes through $x$. Then it is clear that $L_{2, x}$ has the required properties.

Recall, as remarked after equation (2.9), that the natural map

$$
H^{0}\left(C, \mathcal{O}_{C}(d-1)\right) \otimes \mathcal{O}_{\mathcal{Q}}^{n}=p_{\mathcal{Q} *}\left(\mathcal{O}_{C \times \mathcal{Q}}^{n} \otimes p_{C}^{*} \mathcal{O}_{C}(d-1)\right) \rightarrow p_{\mathcal{Q} *}\left(\mathcal{B} \otimes p_{C}^{*} \mathcal{O}_{C}(d-1)\right)
$$

is surjective. Let $\operatorname{Gr}\left(H^{0}\left(C, \mathcal{O}_{C}(d-1)\right)^{n}, d\right)$ denote the Grassmannian of $d$-dimensional quotients of the vector space $H^{0}\left(C, \mathcal{O}_{C}(d-1)\right)^{n}$. Hence we have morphisms

$$
\mathcal{Q} \rightarrow \operatorname{Gr}\left(H^{0}\left(C, \mathcal{O}_{C}(d-1)\right)^{n}, d\right) \hookrightarrow \mathbb{P}^{N},
$$

where the second map is the Plücker embedding. We will denote the first map by $\psi$ and the composition by $\Psi$. Note that, as remarked earlier, we have an isomorphism $\Psi^{*} \mathcal{O}_{\mathbb{P}^{N}}(1) \cong \mathcal{L}_{d-1}$.

Lemma 2.13. The map $\left.\Psi\right|_{\mathcal{U}}: \mathcal{U} \rightarrow \mathbb{P}^{N}$ is injective.

Proof. It is enough to show that the map

$$
\left.\psi\right|_{\mathcal{U}}: \mathcal{U} \rightarrow \operatorname{Gr}\left(H^{0}\left(C, \mathcal{O}_{C}(d-1)\right)^{n}, d\right)
$$


is injective. By definition, if $x \in \mathcal{U}$ corresponds to the quotient $x: \mathcal{O}_{C}^{n} \rightarrow$ $B \rightarrow 0$ then the image of $x$ under the map $\psi$ is the quotient of vector spaces

$$
H^{0}\left(C, \mathcal{O}_{C}(d-1)\right)^{n} \rightarrow H^{0}\left(B \otimes \mathcal{O}_{C}(d-1)\right) \rightarrow 0 .
$$

Let $A=\bigoplus_{i=1}^{n} \mathcal{O}_{C}\left(d_{i}\right)$ be the kernel of $x$. We will show that $A \otimes \mathcal{O}_{C}(d-1)$ is globally generated, which is equivalent to showing $d_{i} \geq-(d-1)$. Let us assume the contrary, that is, there exists $d_{i}$ such that $d_{i} \leq-d$. Then $h^{1}(C, A) \geq h^{1}\left(C, \mathcal{O}_{C}\left(d_{i}\right)\right)=h^{0}\left(C, \mathcal{O}_{C}\left(-2-d_{i}\right)\right) \geq h^{0}\left(C, \mathcal{O}_{C}(-2+d)\right)=d-1$. Now consider the exact sequence

$$
H^{0}\left(C, \mathcal{O}_{C}^{n}\right) \rightarrow H^{0}(C, B) \rightarrow H^{1}(C, A) \rightarrow 0 .
$$

Since $x \in \mathcal{U}$ we have that the image of the first map has dimension $\geq$ 2. Therefore, $h^{1}(C, A) \leq d-2$ and we arrive at a contradiction. Hence $A \otimes \mathcal{O}_{C}(d-1)$ is globally generated. This means that $A$ as a subsheaf of $\mathcal{O}_{C}^{n}$ (and so also the quotient $x$ ) can be recovered from the map $H^{0}(C, A \otimes$ $\left.\mathcal{O}_{C}(d-1)\right) \rightarrow H^{0}\left(C, \mathcal{O}_{C}(d-1)\right)^{n}$ by taking the sheaf generated by the sections $H^{0}\left(C, A \otimes \mathcal{O}_{C}(d-1)\right)$ in $\mathcal{O}_{C}(d-1)^{n}$ and then twisting by $\mathcal{O}_{C}(-d+1)$. Thus, we get that the required map is injective.

Theorem 2.14. Fix integers $a, b>0$. If $x \in \mathcal{U} \subset \mathcal{Q}$ we have

$$
\varepsilon\left(a \cdot\left[\mathcal{L}_{d-1}\right]+b \cdot\left[\Phi^{*} \mathcal{O}_{\mathbb{P}^{d}}(1)\right], x\right)=a .
$$

If $x \in \mathcal{Z}=\mathcal{Q} \backslash \mathcal{U}$ we have

$$
\varepsilon\left(a \cdot\left[\mathcal{L}_{d-1}\right]+b \cdot\left[\Phi^{*} \mathcal{O}_{\mathbb{P}^{d}}(1)\right], x\right)=\min \{a, b\} .
$$

Proof. Let $x \in \mathcal{U}$. Let $X$ be any irreducible and reduced curve in $\mathcal{Q}$ passing through $x$. Then by Lemma 2.13 the map $\Psi(X \cap \mathcal{U}) \neq$ pt. Hence there exists a section $H \in H^{0}\left(\mathcal{Q}, \mathcal{L}_{d-1}\right)$ passing through $x$ such that $X$ is not contained in $H$. By Bézout's theorem, we get

$$
\left[\mathcal{L}_{d-1}\right] \cdot[X]=[H] \cdot[X] \geq \operatorname{mult}_{x} X
$$

Hence

$$
\left(a \cdot\left[\mathcal{L}_{d-1}\right]+b \cdot\left[\Phi^{*} \mathcal{O}_{\mathbb{P}^{d}}(1)\right]\right) \cdot[X] \geq a \cdot\left[\mathcal{L}_{d-1}\right] \cdot[X] \geq a \cdot \operatorname{mult}_{x} X .
$$

Therefore, $\varepsilon\left(a \cdot\left[\mathcal{L}_{d-1}\right]+b \cdot\left[\Phi^{*} \mathcal{O}_{\mathbb{P}^{d}}(1)\right], x\right) \geq a$. Now by Lemma 2.6 we have

$$
\varepsilon\left(a \cdot\left[\mathcal{L}_{d-1}\right]+b \cdot\left[\Phi^{*} \mathcal{O}_{\mathbb{P}^{d}}(1)\right], x\right) \leq \frac{\left(a \cdot\left[\mathcal{L}_{d-1}\right]+b \cdot\left[\Phi^{*} \mathcal{O}_{\mathbb{P}^{d}}(1)\right]\right) \cdot\left[L_{1, x}\right]}{\operatorname{mult}_{x} L_{1, x}}=a .
$$

Hence, we get for $x \in \mathcal{U}$

$$
\varepsilon\left(a \cdot\left[\mathcal{L}_{d-1}\right]+b \cdot\left[\Phi^{*} \mathcal{O}_{\mathbb{P}^{d}}(1)\right], x\right)=a .
$$

Now let $x \in \mathcal{Z}$. We first prove the inequality

$$
\varepsilon\left(a \cdot\left[\mathcal{L}_{d-1}\right]+b \cdot\left[\Phi^{*} \mathcal{O}_{\mathbb{P}^{d}}(1)\right], x\right) \geq \min \{a, b\} .
$$

Let $X$ be any irreducible and reduced curve in $\mathcal{Q}$ passing through $x$. We have maps $\Psi: \mathcal{Q} \rightarrow \mathbb{P} H^{0}\left(\mathcal{Q}, \mathcal{L}_{d-1}\right)$ and $\Phi: \mathcal{Q} \rightarrow \mathbb{P}^{d}$. The class $\left[\mathcal{L}_{d-1}\right]+\left[\Phi^{*} \mathcal{O}_{\mathbb{P}^{d}}(1)\right]$ 
is ample, thus, it cannot happen that $\Psi$ and $\Phi$ both are constant on $X$. First consider the case when $\Psi$ is non-constant on $X$. Hence, there exists a section $H_{1} \in H^{0}\left(\mathcal{Q}, \mathcal{L}_{d-1}\right)$ passing through $x$ such that $X$ is not contained in $H_{1}$. By Bézout's theorem, we will have

$$
\left[\mathcal{L}_{d-1}\right] \cdot[X]=\left[H_{1}\right] \cdot[X] \geq \operatorname{mult}_{x} X
$$

which gives

$$
\frac{\left(a \cdot\left[\mathcal{L}_{d-1}\right]+b \cdot\left[\Phi^{*} \mathcal{O}_{\mathbb{P}^{d}}(1)\right]\right) \cdot[X]}{\operatorname{mult}_{x} X} \geq a \frac{\left[\mathcal{L}_{d-1}\right] \cdot[X]}{\operatorname{mult}_{x} X} \geq a \geq \min \{a, b\} .
$$

Next consider the case when $\Phi$ is non-constant on $X$. Then there is a section $H_{2} \in H^{0}\left(\mathcal{Q}, \Phi^{*} \mathcal{O}_{\mathbb{P} d}(1)\right)$ passing through $x$ such that $X$ is not contained in $\mathrm{H}_{2}$. Again we have by Bézout's theorem

$$
\left[\Phi^{*} \mathcal{O}_{\mathbb{P}^{d}}(1)\right] \cdot[X]=\left[H_{2}\right] \cdot[X] \geq \operatorname{mult}_{x} X,
$$

which gives

$$
\frac{\left(a \cdot\left[\mathcal{L}_{d-1}\right]+b \cdot\left[\Phi^{*} \mathcal{O}_{\mathbb{P}^{d}}(1)\right]\right) \cdot[X]}{\operatorname{mult}_{x} X} \geq b \frac{\left[\Phi^{*} \mathcal{O}_{\mathbb{P}^{d}}(1)\right] \cdot[X]}{\operatorname{mult}_{x} X} \geq b \geq \min \{a, b\} .
$$

Therefore we get

$$
\varepsilon\left(a \cdot\left[\mathcal{L}_{d-1}\right]+b \cdot\left[\Phi^{*} \mathcal{O}_{\mathbb{P}^{d}}(1)\right], x\right) \geq \min \{a, b\} .
$$

Now Lemma 2.6 implies that

$$
\varepsilon\left(a \cdot\left[\mathcal{L}_{d-1}\right]+b \cdot\left[\Phi^{*} \mathcal{O}_{\mathbb{P}^{d}}(1)\right], x\right) \leq \frac{\left(a \cdot\left[\mathcal{L}_{d-1}\right]+b \cdot\left[\Phi^{*} \mathcal{O}_{\mathbb{P}^{d}}(1)\right]\right) \cdot\left[L_{1, x}\right]}{\operatorname{mult}_{x} L_{1, x}}=a .
$$

Similarly Lemma 2.12 implies that

$$
\varepsilon\left(a \cdot\left[\mathcal{L}_{d-1}\right]+b \cdot\left[\Phi^{*} \mathcal{O}_{\mathbb{P}^{d}}(1)\right], x\right) \leq \frac{\left(a \cdot\left[\mathcal{L}_{d-1}\right]+b \cdot\left[\Phi^{*} \mathcal{O}_{\mathbb{P}^{d}}(1)\right]\right) \cdot\left[L_{2, x}\right]}{\operatorname{mult}_{x} L_{2, x}}=b .
$$

Therefore, we get that for $x \in \mathcal{Z}$ we have

$$
\varepsilon\left(a \cdot\left[\mathcal{L}_{d-1}\right]+b \cdot\left[\Phi^{*} \mathcal{O}_{\mathbb{P}^{d}}(1)\right], x\right)=\min \{a, b\} .
$$

This completes the proof of the theorem.

From the above theorem, we immediately obtain the following results.

Corollary 2.15. With the notation as in Theorem 2.14, we have

(1) $\varepsilon\left(a \cdot\left[\mathcal{L}_{d-1}\right]+b \cdot\left[\Phi^{*} \mathcal{O}_{\mathbb{P}^{d}}(1)\right], 1\right)=a$.

(2) $\varepsilon\left(a \cdot\left[\mathcal{L}_{d-1}\right]+b \cdot\left[\Phi^{*} \mathcal{O}_{\mathbb{P}^{d}}(1)\right]\right)=\min \{a, b\}$.

Proof. This is immediate from the definitions of $\varepsilon(L, 1)$ and $\varepsilon(L)$ for a line bundle $L$. 


\section{Seshadri Constants on $\mathcal{Q}(E, d)$ When $d>1$}

We now consider the case of an arbitrary vector bundle $E$ on $C=\mathbb{P}^{1}$. We treat the case $d>1$ in this section. In the next section, we deal with the case $d=1$.

If $E$ is a vector bundle on $C=\mathbb{P}^{1}$, then $E$ is a direct sum of line bundles. By tensoring with a suitable line bundle, we may assume that $E=\mathcal{O}_{C} \oplus$ $\bigoplus_{i=1}^{r-1} \mathcal{O}_{C}\left(a_{i}\right)$ with $0 \leq a_{i} \leq a_{j}$ for $i<j$. Let $\mathcal{Q}^{\prime}:=\mathcal{Q}(E, d)$ be the Quot scheme of torsion quotients of $E$ of degree $d$. First we recall the description of the Nef cone of $\mathcal{Q}^{\prime}$. Let $p_{C}: C \times \mathcal{Q}^{\prime} \rightarrow C$ and $p_{\mathcal{Q}}: C \times \mathcal{Q}^{\prime} \rightarrow \mathcal{Q}^{\prime}$ be the projections. Let $p_{C}^{*} E \rightarrow \mathcal{B}^{\prime} \rightarrow 0$ be the universal quotient over $C \times \mathcal{Q}^{\prime}$. Let $M$ be any line bundle on $C$. By [GS20, Lemma 3.1] the sheaf $p_{\mathcal{Q}^{\prime} *}\left(p_{C}^{*} M \otimes \mathcal{B}^{\prime}\right)$ is a vector bundle over $\mathcal{Q}^{\prime}$ of rank $d$. Define

$$
\mathcal{L}_{M, \mathcal{Q}^{\prime}}:=\operatorname{det}\left(p_{\mathcal{Q}^{\prime} *}\left(p_{C}^{*} M \otimes \mathcal{B}^{\prime}\right)\right) \text {. }
$$

The bundle $\mathcal{L}_{\mathcal{O}, \mathcal{Q}^{\prime}}$ will also be denoted

$$
\mathcal{O}_{\mathcal{Q}^{\prime}}(1):=\mathcal{L}_{\mathcal{O}, \mathcal{Q}^{\prime}}=\operatorname{det}\left(p_{\mathcal{Q}^{\prime} *}\left(\mathcal{B}^{\prime}\right)\right)
$$

Let

$$
L:=\mathcal{O}_{C}(d-1)
$$

Let

$$
\Phi^{\prime}: \mathcal{Q}^{\prime} \rightarrow S^{d} \mathbb{P}^{1} \cong \mathbb{P}^{d}
$$

be the Hilbert-Chow map (see [GS19, Section 2]). Then it is proved in [GS20, Theorem 6.2] that

$$
\operatorname{Nef}\left(\mathcal{Q}^{\prime}\right)=\mathbb{R}_{\geq 0}\left[\mathcal{L}_{L, \mathcal{Q}^{\prime}}\right]+\mathbb{R}_{\geq 0}\left[\Phi^{\prime *} \mathcal{O}_{\mathbb{P}^{d}}(1)\right] .
$$

Lemma 3.6. Given any point $x \in \mathcal{Q}^{\prime}$ there exists a curve $L_{1, x} \cong \mathbb{P}^{1} \hookrightarrow \mathcal{Q}^{\prime}$ passing through $x$ such that $\left[\mathcal{L}_{L, \mathcal{Q}^{\prime}}\right] \cdot\left[L_{1, x}\right]=1$ and $\left[\Phi^{\prime *} \mathcal{O}_{\mathbb{P}^{d}}(1)\right] \cdot\left[L_{1, x}\right]=0$.

Proof. Replace $\mathcal{Q}$ by $\mathcal{Q}^{\prime}$ and proceed exactly as in the proof of Lemma 2.6 till equation (2.10). Thus, we get that $\operatorname{det}\left(p_{\mathcal{Q}^{\prime} *}\left(\mathcal{B}^{\prime} \otimes p_{C}^{*} L\right)\right)=\mathcal{L}_{L, \mathcal{Q}^{\prime}}$ is a globally generated line bundle on $\mathcal{Q}^{\prime}$, and we have a map $f: Z \rightarrow \mathcal{Q}^{\prime}$ such that $\Phi^{\prime} \circ f$ is constant.

In [GS20, Theorem 6.2] it is proved that

$$
\mathcal{L}_{L, \mathcal{Q}^{\prime}} \cong \mathcal{O}_{\mathcal{Q}^{\prime}}(1) \otimes \Phi^{\prime *} \mathcal{O}_{\mathbb{P}^{d}}(d-1) \text {. }
$$

Since $\Phi^{\prime} \circ f$ is constant, it follows that $f^{*} \mathcal{L}_{L, \mathcal{Q}^{\prime}} \cong f^{*} \mathcal{O}_{\mathcal{Q}^{\prime}}(1) \cong \mathcal{O}_{Z}(1)$. Consider the composite map $Z \rightarrow \mathcal{Q}^{\prime} \rightarrow \mathbb{P}^{N}$, where the second map is given by $\mathcal{L}_{L, \mathcal{Q}^{\prime}}$. Since the pullback of $\mathcal{O}_{\mathbb{P}^{N}}(1)$ along this map is $\mathcal{O}_{Z}(1)$ it follows that the composite, and so also $f$, is a closed immersion.

Let $L_{1, x} \subset Z \subset \mathcal{Q}^{\prime}$ denote any line passing through $x$. Then it is clear that $L_{1, x}$ satisfies the assertions of the lemma.

Let $E^{\prime}$ denote the largest proper sub-bundle of $E$ in the Harder-Narasimhan filtration of $E$. Then $E / E^{\prime}$ is the trivial bundle. 
Lemma 3.7. Assume $d>1$. Let $E \rightarrow B$ be a torsion quotient of degree $d$ such that the image of $H^{0}(C, E)$ in $H^{0}(C, B)$ is one-dimensional. Then this quotient factors through $E \rightarrow E / E^{\prime}$.

Proof. Let $V$ denote the vector space $H^{0}(C, E)$. Let $\mathcal{O}(a)$ be a sub-bundle of $E^{\prime}$. Then $a>0$. To show that $\mathcal{O}(a)$ is mapped to 0 by the given projection $E \rightarrow B$, it suffices to show that the global sections are mapped to 0 . If not, then there is a commutative diagram

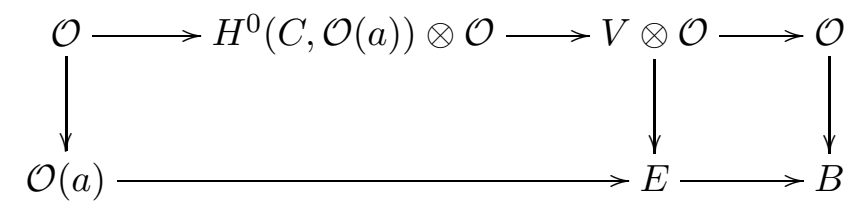

The square on the right exists because of the assumption that the image $H^{0}(C, E) \rightarrow H^{0}(C, B)$ is one-dimensional. By assumption the top horizontal composite map is nonzero. Since the right vertical arrow is a surjection, it follows that the composite map $\mathcal{O}(a) \rightarrow B$ is surjective. We may choose homogeneous coordinates $X$ and $Y$ on $C=\mathbb{P}^{1}$, so that the support of $B$ is contained in the open set $Y \neq 0$. Let $T$ denote the affine coordinate $X / Y$. Thus, the map $\mathcal{O}(a) \rightarrow B$ can be thought of as a surjective map $k[T] \rightarrow k[T] /(p(T))$ of $k[T]$ modules. Viewed like this, a basis for the global sections of $\mathcal{O}(a)$ is given by the functions $T^{i}$ where $0 \leq i \leq a$. The degree of $p(T)$ is equal to the length of the module $k[T] /(p(T)) \cong B$, which is exactly $d$. Since $d>1$, we get a contradiction to the assumption that the image of $H^{0}(C, \mathcal{O}(a))$ is one-dimensional.

There is a natural inclusion $j^{\prime}: \mathcal{Q}\left(E / E^{\prime}, d\right) \hookrightarrow \mathcal{Q}^{\prime}$ which commutes with the Hilbert-Chow maps. Since the bundle $E / E^{\prime}$ is trivial, $\mathcal{Q}\left(E / E^{\prime}, d\right)=$ $\mathcal{Q}\left(n^{\prime \prime}, d\right)=: \mathcal{Q}^{\prime \prime}$. Let $n=\operatorname{dim}\left(H^{0}(C, E)\right)$ and let $\mathcal{Q}:=\mathcal{Q}(n, d)$. The surjection $H^{0}(C, E) \otimes \mathcal{O}_{C} \rightarrow E$ defines an inclusion $j: \mathcal{Q}^{\prime} \hookrightarrow \mathcal{Q}$. The following diagram is commutative.

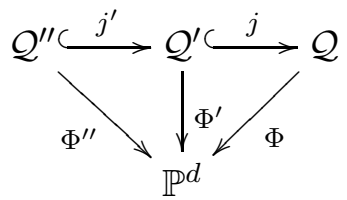

The $\Phi$ 's denote the Hilbert-Chow maps. In particular there are subsets $\mathcal{U}^{\prime \prime} \subset \mathcal{Q}^{\prime \prime}$ and $\mathcal{Z}^{\prime \prime} \subset \mathcal{Q}^{\prime \prime}$ as in Definition 2.11.

Corollary 3.8. Assume $d>1$. Then $\mathcal{Q}^{\prime} \backslash j^{-1}(\mathcal{U})$ is precisely $j^{\prime}\left(\mathcal{Z}^{\prime \prime}\right)$.

Proof. Follows from Lemma 3.7.

Lemma 3.9. Assume $d>1$. Given any point $x \in \mathcal{Q}^{\prime} \backslash j^{-1}(\mathcal{U})$ there exists a curve $L_{2, x} \cong \mathbb{P}^{1} \hookrightarrow \mathcal{Q}^{\prime}$ passing through $x$ such that $\left[\mathcal{L}_{L, \mathcal{Q}^{\prime}}\right] \cdot\left[L_{2, x}\right]=0$ and $\left[\Phi^{\prime \prime *} \mathcal{O}_{\mathbb{P}^{d}}(1)\right] \cdot\left[L_{2, x}\right]=1$. 
Proof. We have maps $\mathcal{Q}^{\prime \prime} \stackrel{j^{\prime}}{\rightarrow} \mathcal{Q}^{\prime} \stackrel{j}{\rightarrow} \mathcal{Q}$. As observed above, $x$ is in the image of $\mathcal{Z}^{\prime \prime}$. It can be shown that $j^{*} \mathcal{L}_{d-1}=\mathcal{L}_{L, \mathcal{Q}^{\prime}}$, for this see the proof of [GS20, Theorem 6.2]. Thus, the Lemma is clear using Lemma 2.12 once we observe that

$$
j^{\prime *} \mathcal{L}_{L, \mathcal{Q}^{\prime}} \cong j^{\prime *} j^{*} \mathcal{L}_{d-1} \cong \mathcal{L}_{d-1, \mathcal{Q}^{\prime \prime}}
$$

This is easily seen using [GS20, Lemma 3.14].

Theorem 3.10. Assume $d>1$. Fix integers $a, b>0$. If $x \in \mathcal{Q}^{\prime}$ such that $j(x) \in \mathcal{U}$, then we have

$$
\varepsilon\left(a \cdot\left[\mathcal{L}_{L, \mathcal{Q}^{\prime}}\right]+b \cdot\left[\Phi^{\prime *} \mathcal{O}_{\mathbb{P}^{d}}(1)\right], x\right)=a .
$$

If $x \in \mathcal{Q}^{\prime} \backslash \mathcal{U}$ we have

$$
\varepsilon\left(a \cdot\left[\mathcal{L}_{L, \mathcal{Q}^{\prime}}\right]+b \cdot\left[\Phi^{\prime *} \mathcal{O}_{\mathbb{P}^{d}}(1)\right], x\right)=\min \{a, b\} .
$$

Proof. Let $x \in \mathcal{Q}^{\prime}$ such that $j(x) \in \mathcal{U}$. Let $X$ be any irreducible and reduced curve in $\mathcal{Q}^{\prime}$ passing through $x$. Consider the composite map

$$
X \hookrightarrow \mathcal{Q}^{\prime} \stackrel{j}{\rightarrow} \mathcal{Q} \stackrel{\Psi}{\rightarrow} \mathbb{P}^{N}
$$

Then by Lemma 2.13 the map $\Psi(j(X) \cap \mathcal{U}) \neq$ pt. Hence there exists a section $H^{0}\left(\mathcal{Q}^{\prime}, \mathcal{L}_{L, \mathcal{Q}^{\prime}}\right)$ whose zero locus $H$ passes through $x$ such that $X$ is not contained in $H$. By Bézout's theorem, we get

$$
\left[\mathcal{L}_{L, \mathcal{Q}^{\prime}}\right] \cdot[X]=[H] \cdot[X] \geq\left(\operatorname{mult}_{x} H\right)\left(\operatorname{mult}_{x} X\right) \geq \operatorname{mult}_{x} X .
$$

Hence

$$
\left(a \cdot\left[\mathcal{L}_{L, \mathcal{Q}^{\prime}}\right]+b \cdot\left[\Phi^{\prime *} \mathcal{O}_{\mathbb{P}^{d}}(1)\right]\right) \cdot[X] \geq a \cdot\left[\mathcal{L}_{L, \mathcal{Q}^{\prime}}\right] \cdot[X] \geq a \cdot \operatorname{mult}_{x} X .
$$

Therefore, $\varepsilon\left(a \cdot\left[\mathcal{L}_{L, \mathcal{Q}^{\prime}}\right]+b \cdot\left[\Phi^{\prime *} \mathcal{O}_{\mathbb{P}^{d}}(1)\right], x\right) \geq a$. Now by Lemma 3.6 we have $\varepsilon\left(a \cdot\left[\mathcal{L}_{L, \mathcal{Q}^{\prime}}\right]+b \cdot\left[\Phi^{\prime *} \mathcal{O}_{\mathbb{P}^{d}}(1)\right], x\right) \leq \frac{\left(a \cdot\left[\mathcal{L}_{L, \mathcal{Q}^{\prime}}\right]+b \cdot\left[\Phi^{\prime *} \mathcal{O}_{\mathbb{P}^{d}}(1)\right]\right) \cdot\left[L_{1, x}\right]}{\operatorname{mult}_{x} L_{1, x}}=a$.

Hence, we get for $x \in \mathcal{U}$

$$
\varepsilon\left(a \cdot\left[\mathcal{L}_{L, \mathcal{Q}^{\prime}}\right]+b \cdot\left[\Phi^{\prime *} \mathcal{O}_{\mathbb{P}^{d}}(1)\right], x\right)=a .
$$

Now let $j(x) \in \mathcal{Z}$. We first prove the inequality

$$
\varepsilon\left(a \cdot\left[\mathcal{L}_{L, \mathcal{Q}^{\prime}}\right]+b \cdot\left[\Phi^{\prime *} \mathcal{O}_{\mathbb{P}^{d}}(1)\right], x\right) \geq \min \{a, b\} .
$$

Let $X$ be any irreducible and reduced curve in $\mathcal{Q}^{\prime}$ passing through $x$. We have maps $\Psi \circ j: \mathcal{Q}^{\prime} \rightarrow \mathcal{Q} \rightarrow \mathbb{P} H^{0}\left(\mathcal{Q}, \mathcal{L}_{d-1}\right)$ and $\Phi^{\prime}: \mathcal{Q}^{\prime} \rightarrow \mathbb{P}^{d}$. The class $\left[\mathcal{L}_{L, \mathcal{Q}^{\prime}}\right]+\left[\Phi^{\prime *} \mathcal{O}_{\mathbb{P d}}(1)\right]$ is ample, thus, it cannot happen that $\Psi$ and $\Phi^{\prime}$ both are constant on $X$. First consider the case when $\Psi$ is non-constant on $X$. Hence, there exists a section $H_{1} \in H^{0}\left(\mathcal{Q}^{\prime}, \mathcal{L}_{L, \mathcal{Q}^{\prime}}\right)$ passing through $x$ such that $X$ is not contained in $H_{1}$. By Bézout's theorem, we will have

$$
\left[\mathcal{L}_{L, \mathcal{Q}^{\prime}}\right] \cdot[X]=\left[H_{1}\right] \cdot[X] \geq \operatorname{mult}_{x} X,
$$

which gives

$$
\frac{\left(a \cdot\left[\mathcal{L}_{L, \mathcal{Q}^{\prime}}\right]+b \cdot\left[\Phi^{\prime *} \mathcal{O}_{\mathbb{P}^{d}}(1)\right]\right) \cdot[X]}{\operatorname{mult}_{x} X} \geq a \geq \min \{a, b\} .
$$


Next consider the case when $\Phi^{\prime}$ is non-constant on $X$. Then there is a section $H_{2} \in H^{0}\left(\mathcal{Q}^{\prime}, \Phi^{\prime *} \mathcal{O}_{\mathbb{P}^{d}}(1)\right)$ passing through $x$ such that $X$ is not contained in $\mathrm{H}_{2}$. Again we have by Bézout's theorem

$$
\left[\Phi^{\prime *} \mathcal{O}_{\mathbb{P}^{d}}(1)\right] \cdot[X]=\left[H_{2}\right] \cdot[X] \geq \operatorname{mult}_{x} X
$$

which gives

$$
\frac{\left(a \cdot\left[\mathcal{L}_{L, \mathcal{Q}^{\prime}}\right]+b \cdot\left[\Phi^{\prime *} \mathcal{O}_{\mathbb{P}^{d}}(1)\right]\right) \cdot[X]}{\operatorname{mult}_{x} X} \geq b \geq \min \{a, b\} .
$$

Therefore we get

$$
\varepsilon\left(a \cdot\left[\mathcal{L}_{L, \mathcal{Q}^{\prime}}\right]+b \cdot\left[\Phi^{\prime *} \mathcal{O}_{\mathbb{P}^{d}}(1)\right], x\right) \geq \min \{a, b\} .
$$

Now Lemma 3.6 implies that

$$
\varepsilon\left(a \cdot\left[\mathcal{L}_{d-1}\right]+b \cdot\left[\Phi^{*} \mathcal{O}_{\mathbb{P}^{d}}(1)\right], x\right) \leq \frac{\left(a \cdot\left[\mathcal{L}_{d-1}\right]+b \cdot\left[\Phi^{*} \mathcal{O}_{\mathbb{P}^{d}}(1)\right]\right) \cdot\left[L_{1, x}\right]}{\operatorname{mult}_{x} L_{1, x}}=a .
$$

Similarly Lemma 3.9 implies that

$$
\varepsilon\left(a \cdot\left[\mathcal{L}_{d-1}\right]+b \cdot\left[\Phi^{*} \mathcal{O}_{\mathbb{P}^{d}}(1)\right], x\right) \leq \frac{\left(a \cdot\left[\mathcal{L}_{d-1}\right]+b \cdot\left[\Phi^{*} \mathcal{O}_{\mathbb{P}^{d}}(1)\right]\right) \cdot\left[L_{2, x}\right]}{\operatorname{mult}_{x} L_{2, x}}=b .
$$

Therefore, we get that for $x \in \mathcal{Z}$ we have

$$
\varepsilon\left(a \cdot\left[\mathcal{L}_{d-1}\right]+b \cdot\left[\Phi^{*} \mathcal{O}_{\mathbb{P}^{d}}(1)\right], x\right)=\min \{a, b\} .
$$

This completes the proof of the theorem.

As before, we immediately obtain the following corollary.

Corollary 3.11. With the notation as in Theorem 3.10, we have

(1) $\varepsilon\left(a \cdot\left[\mathcal{L}_{L, \mathcal{Q}^{\prime}}\right]+b \cdot\left[\Phi^{\prime *} \mathcal{O}_{\mathbb{P} d}(1)\right], 1\right)=a$.

(2) $\varepsilon\left(a \cdot\left[\mathcal{L}_{L, \mathcal{Q}^{\prime}}\right]+b \cdot\left[\Phi^{\prime *} \mathcal{O}_{\mathbb{P} d}(1)\right]\right)=\min \{a, b\}$.

\section{Seshadri COnstants on $\mathcal{Q}(E, 1)$}

Let $Y$ be a smooth projective complex curve and $E$ a vector bundle on $Y$. Let $X=\mathbb{P}(E)$ be the projective bundle associated to $E$ over $Y$. The Quot scheme $\mathcal{Q}(E, 1)$ is isomorphic to $X$. Denote by $\xi:=\mathcal{O}_{\mathbb{P}(E)}(1)$ and by $\mathfrak{f}$ the divisor which is a fibre of $\pi$.

Let $Q$ denote a vector bundle quotient of $E$ with the smallest slope and largest rank. Note that if

$$
0=E_{0} \subset E_{1} \subset \ldots \subset E_{d-1} \subset E_{d}=E
$$

is the Harder-Narasimhan filtration of $E$, then $Q=E / E_{d-1}$. Let $e:=\mu(Q)$ denote the slope of $Q$.

Theorem 4.1 (Miyaoka). The nef cone of $X$ is spanned by $\xi-e \mathfrak{f}$ and $\mathfrak{f}$. 
See [Miy87] and [Ful11, Lemma 2.1]. The dual basis of the closed cone of curves $\overline{N E}(X)$ is given by extremal rays spanned by two 1 -cycles $C_{1}, C_{2}$, where $C_{2}$ denotes the class of a line in a fibre of $\pi$ (which is isomorphic to the projective space $\left.\mathbb{P}^{\mathrm{rk}(E)-1}\right)$. The other 1 -cycle $C_{1}$ is not effective in general. However, $C_{1}$ is the pushforward of a pseudoeffective 1-cycle via the natural embedding $\mathbb{P}(Q) \rightarrow \mathbb{P}(E)$; see [Ful11, Lemma 2.3].

We now assume $Y=\mathbb{P}^{1}$. If $E$ is a trivial vector bundle then $\mathbb{P}(E)=$ $\mathbb{P}^{1} \times \mathbb{P}^{\mathrm{pr}(E)-1}$. In this case, it is easy to compute the Seshadri constants of ample line bundles on $\mathbb{P}(E)$; for example, see [MR15, Proposition 3.4(e)].

Now let $E$ be a bundle such that $E \neq \mathcal{O}^{\operatorname{rk}(E)}$. Since $\mathbb{P}(E) \cong \mathbb{P}(E \otimes L)$ for any line bundle $L$ on $Y$, we may assume $E=\mathcal{O}^{s} \oplus \mathcal{O}\left(a_{1}\right) \ldots \oplus \mathcal{O}\left(a_{r}\right)$ with $0<a_{1} \leq \ldots \leq a_{r}$ (after tensoring $E$ with a suitable line bundle). Then the quotient of $E$ with the smallest slope is $Q=\mathcal{O}^{s}$. In other words,

$$
E_{d-1}=\underset{i \geq 1}{\oplus} \mathcal{O}\left(a_{i}\right)
$$

So we have $e=\mu(Q)=0$. In this case, the extremal ray $C_{1}$, in the above notation, is spanned by the image of the section

$$
\mathbb{P}^{1} \rightarrow X
$$

corresponding to a rank 1 quotient

$$
E \rightarrow \mathcal{O}
$$

Indeed, first observe that $\mathfrak{f} \cdot C_{2}=0$ and $\mathfrak{f} \cdot C_{1}=1$. Next note that $\xi \cdot C_{1}=$ $\operatorname{deg}(\mathcal{O})=0$ and $\xi \cdot C_{2}=1$. So $C_{2}, C_{1}$ is dual to $\xi, \mathfrak{f}$.

We first prove a general result about Seshadri constants on $X$.

Proposition 4.2. Let $L$ be an ample line bundle on $X$ which is numerically equivalent to the bundle $a \xi+b \mathfrak{f}$, where $a, b$ are positive integers. Then $a \geq$ $\varepsilon(X, L, x) \geq \min \{a, b\}$ for all $x \in X$.

Proof. To prove the first inequality, let $x \in X$. Then $x \in \pi^{-1}(\pi(x))=$ $\mathbb{P}^{r+s-1}$. Choose a line $l$ in $\mathbb{P}^{r+s-1}$ containing $x$. The ratio given by this line is $\frac{L \cdot l}{1}=a$. Hence $a \geq \varepsilon(X, L, x)$.

Now we prove the second inequality. Let $C \subset X$ be an irreducible and reduced curve such that $m:=\operatorname{mult}_{x}(C)>0$. Write $C=p C_{1}+q C_{2}$ for non-negative integers $p, q$.

Case 1: Suppose $\pi(C)$ is a point $y \in \mathbb{P}^{1}$. Then $p=0$ and $C$ is a curve in $\pi^{-1}(y)$ of degree $q$. Thus, $C \subset \mathbb{P}^{r+s-1}$ and if $H$ denotes a general hyperplane through $x$ then

$$
q=C \cdot H \geq\left(\operatorname{mult}_{x} C\right)\left(\operatorname{mult}_{x} H\right) \geq m
$$

So

$$
\frac{L \cdot C}{m}=\frac{a q}{m} \geq a \geq \min \{a, b\}
$$


Case 2: Suppose $\pi(C)=\mathbb{P}^{1}$. Let $W=\pi^{-1}(\pi(x))$. Since $C \not \subset W$, Bézout's theorem gives

$$
p=\mathfrak{f} \cdot C=W \cdot C \geq\left(\operatorname{mult}_{x} C\right)\left(\operatorname{mult}_{x} W\right) \geq m .
$$

Hence

$$
\frac{L \cdot C}{m}=\frac{a q+b p}{m} \geq b \geq \min \{a, b\} .
$$

So $\frac{L \cdot C}{m} \geq \min \{a, b\}$ for all curves $C$ passing through $x$. This gives the desired bound $\varepsilon(X, L, x) \geq \min \{a, b\}$ for all $x \in X$.

Now we obtain more precise values of the Seshadri constants on $X$. Recall that $E / E_{d-1}=\mathcal{O}^{s}$. We get a morphism $i: \mathbb{P}\left(E / E_{d-1}\right) \hookrightarrow X$ using the quotient

$$
E \rightarrow E / E_{d-1} \rightarrow \mathcal{O}_{\mathbb{P}\left(E / E_{d-1}\right)}(1) .
$$

Let $Z$ denote the image of $i$. We have $Z \cong \mathbb{P}^{1} \times \mathbb{P}^{s-1}$ and $i^{*} \xi=p_{2}^{*} \mathcal{O}_{\mathbb{P}^{s-1}}(1)$ and $i^{*} \mathfrak{f}=p_{1}^{*} \mathcal{O}_{\mathbb{P}^{1}}(1)$ where $p_{i}$ are the projections from $\mathbb{P}^{1} \times \mathbb{P}^{s-1}$.

Theorem 4.3. Let $L$ be an ample line bundle on $X$ which is numerically equivalent to the bundle $a \xi+b \mathfrak{f}$, where $a, b$ are positive integers. Let $x \in X$. We have

$$
\varepsilon(L, x)= \begin{cases}a, & \text { if } x \notin Z, \\ \min \{a, b\}, & \text { if } x \in Z .\end{cases}
$$

Proof. Suppose first that $x \in Z$. The restriction of $L$ to $Z$ is the bundle $L_{Z}:=p_{1}^{*} \mathcal{O}_{\mathbb{P}^{1}}(a) \otimes p_{2}^{*} \mathcal{O}_{\mathbb{P}^{s-1}}(b)$. It is easy to see that $\varepsilon\left(Z, L_{Z}, x\right)=\min \{a, b\}$ (for example, see [MR15, Proposition 3.4(e)]). Obviously $\varepsilon(X, L, x) \leq$ $\varepsilon\left(Z, L_{Z}, x\right)$. By Proposition 4.2 , it follows that $\varepsilon(X, L, x)=\min \{a, b\}$.

Now assume $x \notin Z$. Let $C \subset X$ be an irreducible and reduced curve such that $m:=\operatorname{mult}_{x}(C)>0$. So we have $C \not \subset Z$. Let $\phi: C \rightarrow \mathbb{P}^{1}$ be the composition of the inclusion $C \subset X$ with the natural map $X \rightarrow \mathbb{P}^{1}$. Let $\phi^{*} E \rightarrow M$ be the line bundle quotient which defines the map $C \rightarrow$ $\mathbb{P}(E)$. Then since $C$ is not contained in $Z$, the quotient map $\phi^{*} E \rightarrow M$ does not factor through $\phi^{*} E / \phi^{*} E_{d-1}$. In other words, the composition map $\phi^{*} E_{d-1} \rightarrow \phi^{*} E \rightarrow M$ is not zero. Recall $E_{d-1}=\underset{i \geq 1}{\oplus} \mathcal{O}\left(a_{i}\right)$ and all $a_{i}>0$. Thus, the composition $\phi^{*} \mathcal{O}\left(a_{i}\right) \subset \phi^{*} E_{d-1} \rightarrow M$ is non-zero for some $i$. Write $C=p C_{1}+q C_{2}$ for non-negative integers $p, q$. Note that $p=C \cdot \mathfrak{f}=\operatorname{deg}(\phi)$. Thus, we get

$$
q=\xi \cdot C=\operatorname{deg}\left(\left.\xi\right|_{C}\right)=\operatorname{deg}(M) \geq a_{i} \operatorname{deg}(\phi)=a_{i} p \geq p .
$$

Suppose that $\pi(C)$ is a point in $\mathbb{P}^{1}$. Then by Case 1 of Proposition 4.2 , we have

$$
\frac{L \cdot C}{m}=\frac{a q}{m} \geq a
$$


Next suppose that $\pi(C)=\mathbb{P}^{1}$. Then arguing as in Case 2 of Proposition 4.2 , we conclude $p \geq m$. So $q \geq p \geq m$ and

$$
\frac{L \cdot C}{m}=\frac{a q+b p}{m} \geq \frac{(a+b) p}{m} \geq a+b \geq a .
$$

So $\frac{L \cdot C}{m} \geq a$ for all curves $C$ passing through $x$. This gives $\varepsilon(X, L, x) \geq a$.

On the other hand, let $W=\pi^{-1}(\pi(x))$. Let $D \subset W \cong \mathbb{P}^{r-1}$ be a line containing $x$. Then $D$ is smooth and $L \cdot D=a$. So $\varepsilon(X, L, x) \leq a$.

We conclude that $\varepsilon(X, L, x)=a$ for all $x \notin Z$, as required.

Corollary 4.4. With the notation as in Theorem 4.3, we have

(1) $\varepsilon(L, 1)=a$.

(2) $\varepsilon(L)=\min \{a, b\}$.

Remark 4.5. If $s=1$ in the above notation, Theorem 4.3 follows directly from [BHNN20, Theorem 3.3]. If $s=1$ then the rank of $E_{d} / E_{d-1}$ is 1 . We take $m=d, r=1$ in the notation of [BHNN20, Section 2.1]. So the Grassmann bundle is nothing but the projective bundle $\mathbb{P}(E)$. Then the sub variety $Z=\mathbb{P}\left(E / E_{d-1}\right) \subset X$ defined above coincides with the section $\Gamma_{s}$ defined in [BHNN20, Section 3]. The other hypotheses of [BHNN20, Theorem 3.3] can be verified easily to obtain Theorem 4.3.

\section{Seshadri constants on $G r(E, n)$}

In this section, we compute Seshadri constants for ample line bundles on the Grassmann bundle over $C=\mathbb{P}^{1}$. For a vector bundle $E$ on $\mathbb{P}^{1}$ and a positive integer $n$, recall that $G r(E, n)$ denotes the Grassmann bundle that parametrizes the $n$-dimensional quotients of the fibers of $E$.

Given a vector bundle $E$ on $\mathbb{P}^{1}$, we tensor $E$ with a suitable line bundle and assume that $E=\mathcal{O}^{r_{0}} \oplus \mathcal{O}\left(a_{1}\right)^{r_{1}} \oplus \ldots \oplus \mathcal{O}\left(a_{m}\right)^{r_{m}}$, with $0<a_{1}<\ldots<a_{m}$ and $r_{i}>0$ for all $0 \leq i \leq m$. Define $a_{0}=0$ and $r_{-1}=0$. The least possible degree among all possible quotients of $E$ of rank $n$ is described as follows. Let $t$ be the smallest integer such that

$$
r_{0}+\ldots+r_{t} \geq n \text {. }
$$

Define $l:=r_{0}+\ldots+r_{t-1}$; then $l<n$. Then the quotient of $E$ of least possible degree is isomorphic to

$$
\left(\bigoplus_{i=0}^{t-1} \mathcal{O}\left(a_{i}\right)^{r_{i}}\right) \oplus \mathcal{O}\left(a_{t}\right)^{n-l}
$$

Clearly, this has degree $d_{0}:=(n-l) a_{t}+\sum_{i=1}^{t-1} r_{i} a_{i}$. Consider the Plücker embedding $i: G r(E, n) \subset \mathbb{P}\left(\wedge^{n} E\right)$. Let $\pi: \mathbb{P}\left(\wedge^{n} E\right) \rightarrow \mathbb{P}^{1}$ denote the projection and let $\pi^{\prime}:=\pi \circ i$. Then the above quotient defines a section $s$ of $\pi^{\prime}$ and $i \circ s$ defines a section of $\pi$. One checks easily that the nef cone of $\mathbb{P}\left(\wedge^{n} E\right)$ has as boundaries $\mathfrak{f}=\pi^{*} \mathcal{O}_{\mathbb{P}^{1}}(1)$ and $\xi=\mathcal{O}_{\mathbb{P}\left(\wedge^{n} E\right)}(1) \otimes \pi^{*} \mathcal{O}_{\mathbb{P}^{1}}\left(-d_{0}\right)$. The latter line bundle is strictly nef as $\left(\wedge^{n} E\right) \otimes \mathcal{O}_{\mathbb{P}^{1}}\left(-d_{0}\right)$ is globally generated and has the trivial bundle as a quotient. Using the section $s$ one easily 
checks that the nef cone of $\mathbb{P}\left(\wedge^{n} E\right)$ maps onto the nef cone of $\operatorname{Gr}(E, n)$. See [BP14] for a description of the Nef cones of flag varieties over any curve.

Let $Z:=\operatorname{Gr}\left(\mathcal{O}\left(a_{t}\right)^{r_{t}}, n-l\right)$. Let $\pi^{\prime \prime}: Z \rightarrow \mathbb{P}^{1}$ denote the canonical map. Then we have the tautological quotient

$$
q_{Z}: \pi^{\prime \prime *} \mathcal{O}\left(a_{t}\right)^{r_{t}} \rightarrow F_{Z}
$$

on $Z$. Denote by $E^{\prime}$ and $E^{\prime \prime}$ the following summands of $E$,

$$
E^{\prime}=\bigoplus_{i=0}^{t-1} \mathcal{O}\left(a_{i}\right)^{r_{i}} \quad \text { and } \quad E^{\prime \prime}=\bigoplus_{i=t+1}^{l} \mathcal{O}\left(a_{i}\right)^{r_{i}} .
$$

Thus, $E=E^{\prime} \oplus \mathcal{O}\left(a_{t}\right)^{r_{t}} \oplus E^{\prime \prime}$. We get the following quotient on $Z$

$$
\pi^{\prime \prime *} E=\pi^{\prime \prime *} E^{\prime} \oplus \pi^{\prime \prime *} \mathcal{O}\left(a_{t}\right)^{r_{t}} \oplus \pi^{\prime \prime *} E^{\prime \prime} \stackrel{I d \oplus q_{Z} \oplus 0}{\longrightarrow} \pi^{\prime \prime *} E^{\prime} \oplus F_{Z} \oplus 0,
$$

which defines a map $Z \rightarrow G r(E, n)$.

Lemma 5.1. Let $C \subset G r(E, n)$ be a curve such that $\xi \cdot C=0$. Then this inclusion factors as $C \rightarrow Z \rightarrow G r(E, n)$.

Proof. Let $\phi: C \rightarrow \mathbb{P}^{1}$ denote the projection to $\mathbb{P}^{1}$. Let $q: \phi^{*} E \rightarrow Q$ denote the quotient which defines the map $C \rightarrow G r(E, n)$. Note that if $a: Q \rightarrow Q$ is an isomorphism, then the quotients $q$ and $a \circ q$ define the same maps from $C \rightarrow \operatorname{Gr}(E, n)$. We will find an $a$ for which it is clear that the map defined by $a \circ q$ factors through $Z$.

Since $\xi \cdot C=0$ it is clear that $\operatorname{deg}(\operatorname{det}(Q))=d_{0} \operatorname{deg}(\phi)$. The bundle $\phi^{*}\left(\wedge^{n} E\right)$ is a direct sum of line bundles, each of which has degree at least $d_{0} \operatorname{deg}(\phi)$. Since a line bundle cannot map to a line bundle of strictly lower degree, it follows that there is a line bundle summand of $\phi^{*}\left(\wedge^{n} E\right)$ of degree $d_{0} \operatorname{deg}(\phi)$ which maps isomorphically onto $\operatorname{det}(Q)$. From this we easily conclude that $Q \cong \phi^{*} E^{\prime} \oplus \phi^{*} \mathcal{O}\left(a_{t}\right)^{n-l}$ and that there is a summand of $\phi^{*} E$ such that the composite

$$
\phi^{*} E^{\prime} \oplus \phi^{*} \mathcal{O}\left(a_{t}\right)^{n-l} \subset \phi^{*} E \stackrel{q}{\rightarrow} \phi^{*} E^{\prime} \oplus \phi^{*} \mathcal{O}\left(a_{t}\right)^{n-l}
$$

is an isomorphism. Denote the above isomorphism by $a$. By looking at degree it is clear that the summand $\phi^{*} E^{\prime \prime} \subset \phi^{*} E$ maps to 0 under $q$. Consider the map $a^{-1} \circ q$. It follows that there is a quotient

$$
q^{\prime}: \phi^{*} \mathcal{O}\left(a_{t}\right)^{r_{t}} \rightarrow \phi^{*} \mathcal{O}\left(a_{t}\right)^{n-l}
$$

such that $a^{-1} \circ q$ is of the type

$$
\phi^{*} E=\phi^{*} E^{\prime} \oplus \phi^{*} \mathcal{O}\left(a_{t}\right)^{r_{t}} \oplus \phi^{*} E^{\prime \prime} \stackrel{I d \oplus q^{\prime} \oplus 0}{\longrightarrow} \phi^{*} E^{\prime} \oplus \phi^{*} \mathcal{O}\left(a_{t}\right)^{n-l} \oplus 0 .
$$

The quotient $q^{\prime}$ defines a map $C \rightarrow Z$ and it is clear that the map $C \rightarrow$ $\operatorname{Gr}(E, n)$ factors as $C \rightarrow Z \rightarrow G r(E, n)$. 
Theorem 5.2. Let $L$ be an ample line bundle on $\operatorname{Gr}(E, n)$ which is numerically equivalent to the bundle $a \xi+b \mathfrak{f}$, where $a, b$ are positive integers. Let $x \in \operatorname{Gr}(E, n)$. We have

$$
\varepsilon(L, x)= \begin{cases}a, & \text { if } x \notin Z, \\ \min \{a, b\}, & \text { if } x \in Z .\end{cases}
$$

Proof. If $x \notin Z$ then for each curve $C$ through $x$ we have $\xi \cdot C>0$. Since $\xi$ is a globally generated line bundle we have a map $\psi: G r(E, n) \rightarrow$ $\mathbb{P} H^{0}(G r(E, n), \xi)$. Let $C$ be a curve through $x$. Then since $\xi \cdot C>0$ it follows that $\psi(C)$ is a curve passing through $\psi(x)$. There is a hyperplane through $\psi(x)$ such that the intersection with $\psi(C)$ is proper. Thus, there is a global section of $\xi$ whose vanishing locus is a divisor $H$ through $x$ and its intersection with $C$ is proper. This shows, using Bézout's theorem, that

$$
\xi \cdot C \geq \operatorname{mult}_{x}(H) \operatorname{mult}_{x}(C) \geq \operatorname{mult}_{x}(C) .
$$

Thus,

$$
\frac{(a \xi+b \mathfrak{f}) \cdot C}{\operatorname{mult}_{x}(C)} \geq a
$$

for all curves $C$ passing through $x$. By taking $C$ to be a line in the fiber of $\pi^{\prime}$ we see that the ratio $a$ is attained. This shows that

$$
\varepsilon(G r(E, n), a \xi+b \mathfrak{f}, x)=a .
$$

Next consider the case when $x \in Z$. Note that $Z \cong \mathbb{P}^{1} \times \operatorname{Gr}\left(r_{t}, n-l\right)$. It is easily checked that the restriction of $\xi$ to $Z$ is $\mathcal{O}_{\operatorname{Gr}\left(r_{t}, n-l\right)}(1)$. Thus, through every point of $Z$ there is a section $s$ of $\pi^{\prime}$ such that $\xi \cdot s\left(\mathbb{P}^{1}\right)=0$ and $\mathfrak{f} \cdot s\left(\mathbb{P}^{1}\right)=1$. Thus, the ratio

$$
\frac{(a \xi+b \mathfrak{f}) \cdot s\left(\mathbb{P}^{1}\right)}{\operatorname{mult}_{x}\left(s\left(\mathbb{P}^{1}\right)\right)}=b
$$

is attained. By Theorem 4.3

$$
\varepsilon(G r(E, n), a \xi+b \mathfrak{f}, x) \geq \varepsilon\left(\mathbb{P}\left(\wedge^{n} E\right), a \xi+b \mathfrak{f}, x\right) \geq \min \{a, b\} .
$$

This shows that $\varepsilon(\operatorname{Gr}(E, n), a \xi+b \mathfrak{f}, x)=b$. This completes the proof of the theorem.

Corollary 5.3. With the notation as in Theorem 5.2, we have

(1) $\varepsilon(L, 1)=a$.

(2) $\varepsilon(L)=\min \{a, b\}$.

Remark 5.4. Seshadri constants on Grassmann bundles $G r(E, n)$ over arbitrary smooth curves are studied in [BHNN20]. However, they only consider Grassmann bundles corresponding to rank $n$ quotients for certain specific values of $n$, which are determined by the Harder-Narasimhan filtration of $E$. In Theorem 5.2, we do not impose any conditions on $n$ and determine Seshadri constants for any line bundle on a Grassmann bundle over $\mathbb{P}^{1}$. 


\section{REFERENCES}

[Bau98] Thomas Bauer. Seshadri constants and periods of polarized abelian varieties. Math. Ann., 312(4):607-623, 1998. doi:10.1007/s002080050238. With an appendix by the author and Tomasz Szemberg.

[BHNN20] Indranil Biswas, Krishna Hanumanthu, Donihakkalu Shankar Nagaraj, and Peter E. Newstead. Seshadri constants and grassmann bundles over curves. Annales of the Fourier Institute, 70(4):1477-1496, 2020. doi:10.5802 / aif.3370.

[BP14] Indranil Biswas and A. J. Parameswaran. Nef cone of flag bundles over a curve. Kyoto J. Math., 54(2):353-366, 2014. doi:10.1215/21562261-2642422.

[BS09] Thomas Bauer and Tomasz Szemberg. Seshadri constants and the generation of jets. J. Pure Appl. Algebra, 213(11):2134-2140, 2009. doi:10.1016/j.jpaa.2009.03.005.

[Dem92] Jean-Pierre Demailly. Singular Hermitian metrics on positive line bundles. In Complex algebraic varieties (Bayreuth, 1990), volume 1507 of Lecture Notes in Math., pages 87-104. Springer, Berlin, 1992. doi:10.1007/BFb0094512.

[EL93] Lawrence Ein and Robert Lazarsfeld. Seshadri constants on smooth surfaces. Number 218, pages 177-186. 1993. Journées de Géométrie Algébrique d'Orsay (Orsay, 1992).

[Ful11] Mihai Fulger. The cones of effective cycles on projective bundles over curves. Math. Z., 269(1-2):449-459, 2011. doi:10.1007/s00209-010-0744-z.

[GS19] Chandranandan Gangopadhyay and Ronnie Sebastian. Fundamental group schemes of some Quot schemes on a smooth projective curve. Journal of Algebra, 562:290-305, 2019, arXiv:1909.10775. doi:10.1016/j.jalgebra.2020.06.025.

[GS20] Chandranandan Gangopadhyay and Ronnie Sebastian. Nef cones of some quot schemes on a smooth projective curve, 2020, arXiv:2006.16666.

[Har70] Robin Hartshorne. Ample subvarieties of algebraic varieties. Lecture Notes in Mathematics, Vol. 156. Springer-Verlag, Berlin-New York, 1970. Notes written in collaboration with C. Musili.

[Ito14] Atsushi Ito. Seshadri constants via toric degenerations. J. Reine Angew. Math., 695:151-174, 2014. doi:10.1515/crelle-2012-0116.

[Laz96] Robert Lazarsfeld. Lengths of periods and Seshadri constants of abelian varieties. Math. Res. Lett., 3(4):439-447, 1996. doi:10.4310/MRL.1996.v3.n4.a1.

[Laz04] Robert Lazarsfeld. Positivity in algebraic geometry. I, volume 48 of Ergebnisse der Mathematik und ihrer Grenzgebiete. 3. Folge. A Series of Modern Surveys in Mathematics [Results in Mathematics and Related Areas. 3rd Series. A Series of Modern Surveys in Mathematics]. Springer-Verlag, Berlin, 2004. doi:10.1007/978-3-642-18808-4. Classical setting: line bundles and linear series.

[Lee03] Seunghun Lee. Seshadri constants and Fano manifolds. Math. Z., 245(4):645656, 2003. doi:10.1007/s00209-003-0561-8.

[LZ18] Yuchen Liu and Ziquan Zhuang. Characterization of projective spaces by Seshadri constants. Math. Z., 289(1-2):25-38, 2018. doi:10.1007/s00209-017-1941-9.

[Miy87] Yoichi Miyaoka. The Chern classes and Kodaira dimension of a minimal variety. In Algebraic geometry, Sendai, 1985, volume 10 of $A d v$. Stud. Pure Math., pages 449-476. North-Holland, Amsterdam, 1987. doi:10.2969/aspm/01010449.

[MR15] David McKinnon and Mike Roth. Seshadri constants, diophantine approximation, and Roth's theorem for arbitrary varieties. Invent. Math., 200(2):513-583, 2015. doi:10.1007/s00222-014-0540-1.

[Nak96] Michael Nakamaye. Seshadri constants on abelian varieties. Amer. J. Math., 118(3):621-635, 1996. URL http://muse.jhu.edu/journals/american_journal_of_mathematics/v118/118.3nakamaye.pdf. 
[Str87] Stein Arild Stromme. On parametrized rational curves in Grassmann varieties. In Space curves (Rocca di Papa, 1985), volume 1266 of Lecture Notes in Math., pages 251-272. Springer, Berlin, 1987. doi:10.1007/BFb0078187.

Department of Mathematics, Indian Institute of Technology Bombay, Powai, Mumbai 400076, Maharashtra, India.

Email address: chandra@math.iitb.ac.in

Chennai Mathematical Institute, H1 SipCOT IT Park, Siruseri, KelamBAKKAM 603103, INDIA

Email address: krishna@cmi.ac.in

Department of Mathematics, Indian Institute of Technology Bombay, Powai, Mumbai 400076, Maharashtra, India.

Email address: ronnie@math.iitb.ac.in 